\title{
Schätzen Entscheider im Gesundheitswesen die Niederlassungssituation falsch ein?
}

\author{
Motivation von Ärztinnen und Ärzten zur Niederlassung in eine Hausarzt-Praxis \\ im Bereich der Kassenärztlichen Vereinigung Nordrhein
}

\section{Do decision-makers in the German healthcare system misjudge the situation of a branch office?}

\author{
Motivation of physicians to set up in a GP's practice in the area of the Association \\ of Statutory Health Insurance Physicians North-Rhine
}

\section{Autoren}

Marcus Redaèlli ${ }^{*}$, Maren Cizmowski ${ }^{2}$, Horst Christian Vollmar ${ }^{3}$, Miguel Tamayo $^{4}$, Arim Shukri ${ }^{1}$, Stephanie Stock ${ }^{1}$, August Wilhelm Bödecker ${ }^{2}$

\section{(ㄷ) (1) $(8)$}

Institute

1 Institut für Gesundheitsökonomie und klinische Epidemiologie, Universitätsklinik Köln, Köln

2 Schwerpunkt Allgemeinmedizin am Zentrum für Versorgungsforschung, Universitätsklinik Köln, Köln

3 Abteilung für Allgemeinmedizin, Ruhr-Universität Bochum, Bochum

4 Kassenärztliche Vereinigung Nordrhein, Abt. Medizin und Versorgung, Düsseldorf

Schlüsselwörter

Ärztemangel, Niederlassung, Motivation, Befragung, Versorgungsforschung

Key words

shortage of physicians, branch, motivation, survey, health services research

Bibliografie

DOI https://doi.org/10.1055/a-1034-5963

Online-Publikation: 2.3.2020

Dtsch Med Wochenschr 2020; 145: e50-e60

(c) Georg Thieme Verlag KG, Stuttgart · New York

ISSN 0012-0472

Korrespondenzadresse

Dr. Marcus Redaèlli

Institut für Gesundheitsökonomie und klinische

Epidemiologie

Universitätsklinik Köln, Gleueler Straße 176-178, 50935 Köln marcus.redaelli@uk-koeln.de

Beide Autoren sind Erstautoren.

\section{ZUSAMMENFASSUNG}

Hintergrund Ziel der Untersuchung war die Abbildung von Motiven zur hausärztlichen Niederlassung aus der aktuellen Sicht nach Niederlassung (Erwartungen erfüllt) sowie vor Niederlassung (Motivation). Zudem sollten hinderliche bzw. förderliche Faktoren einer Niederlassung identifiziert werden. Methoden Mittels Mixed-Methods-Ansatz (Fokusgruppen, Einzelinterviews) der Zielgruppe wurden Erfahrungen, Meinungen und Motivationen zur hausärztlichen Niederlassung erfasst, anschließend wurde ein prä-getesteter Fragebogen eingesetzt. Eine Vollerhebung von 675 identifizierten Hausärzten aus der Datenbank der Kassenärztlichen Vereinigung Nordrhein wurde durchgeführt. Das Einschlusskriterium war: Niederlassung 5 Jahren vor Befragungszeitraum (09/2015). Der Fragebogen enthielt 17 Fragen zur Niederlassungsmotivation, 11 zu biografischen Aspekten.

Ergebnisse Die Anzahl der auswertbaren Datensätze betrug 437 (64,7\%). Die höchsten Zustimmungswerte, im Sinne von förderlich für die Niederlassungsmotivation, erhielten „Wegfall der Dienste im Krankenhaus“ (97,2\%) und „eigener Chef sein“ (96,2\%). Als eher hinderlich wurden „Rechte und Pflichten als Vertragsarzt“ (8,5\%) und „vertragsärztlicher Notdienst/Bereitschaftsdienst“ (22,7\%) wahrgenommen. Im Sinne der Erfüllung von Erwartungen stellten sich „Wegfall der Dienste“ (95,6\%) und „Vielfalt der zu behandelnden Altersgruppen“ $(88,9 \%)$ heraus. Insgesamt würden sich $97 \%$, unabhängig vom Erfüllungsgrad ihrer Wünsche, wieder niederlassen und fast 3 Viertel entschieden sich erst nach dem Studium für die hausärztliche Tätigkeit.

Schlussfolgerungen Die Tatsache, dass die Flucht aus dem stationären Sektor als Hauptantrieb zur Niederlassung zählt, muss nachdenklich stimmen. Da dieser Befragungsansatz eine Vollerhebung von hausärztlich Niedergelassenen aus einem 5-jährigen Zeitraum darstellt, kann festgestellt werden, dass aufgrund der Altersstruktur der Befragten die 
derzeitigen Anreizstrukturen einer Niederlassung neu überdacht werden sollten.

\section{ABSTRACT}

Background The aim of the study was to illustrate motives for a GP's branch from the current point of view by branch (expectations fulfilled) and before branch (motivation). In addition, obstructive or beneficial factors of a branch should be identified.

Methods Using a mixed-methods approach (focus groups, individual interviews) with the target group were used in the qualitative part of experiences, opinions and motivations for the GP's branch and, after evaluation, a pre-tested questionnaire was used. A full survey of 675 identified GPs from the database of the Association of Statutory Health Insurance Physicians North Rhine was carried out. These had settled in the last 5 years before the survey period (09/2015). The questionnaire sent by post contained 17 questions on settlement motivation and 11 biographical aspects.
Results The number of evaluable data records was 437 $(64.7 \%)$. The highest approval values, in the sense of conducive to settlement motivation, were "discontinuation of services in the hospital" (97.2\%) and "being one's own boss" (96.2\%). Rights and duties as a "panel physician" (8.5\%), and the "emergency service for panel physicians/on-call service" (22.7\%) were perceived as more of an obstacle. In terms of fulfilling expectations, the "discontinuation of services" (95.6\%) and the "diversity of age groups to be treated" $(88.9 \%)$ emerged. A total of $97 \%$ would settle down again, regardless of the degree to which their wishes were fulfilled, and almost $3 / 4$ would only decided to work as family doctors after they had completed their studies.

Conclusions The fact that escaping from the stationary sector is the main driving force behind the settlement must be thought-provoking. Since this survey approach was not based on specialist groups or the level of training, but on a complete survey of established persons over a five-year period, it can be stated that the current incentive structures of a branch should be rethought due to the age structure of the interviewees.

\section{Einleitung}

Der seit fast 1 Jahrzehnt angekündigte Fachärztemangel ist allenthalben sichtbar, vor allem in der hausärztlichen Versorgung [1]. Besonders betroffen sind die Flächenländer, die mit unterschiedlichen Ansätzen versuchen, Hausärzte zur Niederlassung in unterversorgten bzw. von Unterversorgung bedrohten Gebieten zu bewegen. Eine nachhaltige Veränderung ist jedoch schwierig zu bewerkstelligen, da das Gesundheitssystem jahrzehntelang auf Eindämmung der Kosten und der Anzahl niedergelassener Ärzte ausgerichtet war.

Eine internationale Literaturanalyse identifizierte die Rekrutierung von Medizinstudierenden aus ländlichen Regionen sowie landarztspezifische Unterrichtsangebote in Aus- und Weiterbildung als wirksame Instrumente, Versorgungslücken auf dem Land vorzubeugen [2]. Aufgrund der langen Ausbildungszeit ist ein Effekt derartiger Maßnahmen erst Jahre nach deren Einführung zu erwarten. Vorstöße wie die des nordrhein-westfälischen Gesundheitsministers zur Einführung einer Landarztquote können erst Ende der 2020er-Jahre auf ihre Wirksamkeit überprüft werden.

Es fehlt die Antwort, warum ein Arzt sich dort niederlassen soll, wo Pfarrer und Notare sich bereits verabschiedet haben. Fehlende Infrastruktur für Kinder und Partner verleidet vielfach die Niederlassung auf dem Land. Zudem haben veränderte Lebenswelten, hier vor allem die „Work-Life-Balance“ und die Feminisierung des Berufsstandes, dazu beigetragen, dass ein verändertes Selbstverständnis der heutigen Ärztegenerationen entstanden ist.

Der gesellschaftliche Wandel hat sich auch im deutschen Gesundheitswesen niedergeschlagen [3]. Schlagwörter wie Demografie, medizinischer und technologischer Fortschritt oder Feminisierung der Ärzteschaft prägen die Diskussion um den Ärztemangel [4]. In den wenigsten Studien wurden allerdings diejenigen befragt, die eine Entscheidung für die hausärztliche
Niederlassung getroffen haben. Dabei liegt es auf der Hand, dass diese Gruppe am besten darüber Auskunft geben kann, welche Motive sie zu dieser Entscheidung bewogen haben.

Um eine Bestandsaufnahme von Hausärzten und deren Niederlassungsmotivation abzubilden, führte der Schwerpunkt Allgemeinmedizin des Universitätsklinikums Köln mit Unterstützung der Kassenärztlichen Vereinigung Nordrhein die vorliegende Untersuchung durch.

\section{Methodik}

Das Ziel dieser Untersuchung war die Erhebung von Motiven für eine Niederlassung als Hausarzt sowie eine subjektive Bewertung dieser Motive und weiterer Rahmenbedingungen als fördernde oder hemmende Faktoren. Als methodischer Ansatz wurde das Mixed-Methods-Design verwendet. Hier wurde nach dem qualitativen Anteil ein Fragebogen entwickelt, der persönliche Gründe (wie z. B. freie Zeiteinteilung) und strukturelle Anreize (wie z. B. Wegfall von Diensten im Krankenhaus) abbilden soll. Dabei wurden alle Motive aus 3 Perspektiven abgefragt: es wurde nach Gründen gefragt, die vor der Niederlassung lagen (Initialzündung), nach förderlichen und hinderlichen Gründen während der Niederlassungsphase und ob die Erwartungen nach der Niederlassung erfüllt wurden. Als Antwortkategorien wurde für die Initialzündung eine 4-stufige Likert-Skala verwendet, für die hinderlichen oder förderlichen Faktoren sollte dichotom geantwortet werden und für die Abfrage zur Erfüllung der Erwartungen kam eine 5-stufige Likert-Skala zum Einsatz. Zur Einordnung der Antworten wurden zudem Daten zur persönlichen und beruflichen Biografie erhoben.

Im Vorfeld war eine Literatursichtung zu früheren Untersuchungen durchgeführt worden. Dabei wurden beispielsweise Analysen zur Motivation einer Aufnahme des Humanmedizinstu- 
diums, zum Wandel des Berufsbilds, Ansehen des Hausarztes oder Analysen des Hausarztmangels ausgewertet [5-13]. Zudem fanden 2 Fokusgruppen und Einzelinterviews mit niederlassungswilligen Ärzten aus den Kliniken, Ärzten in der Weiterbildungsphase aus Kliniken und Praxen sowie Hausärzten, die nicht länger als 3 Jahre niedergelassen waren, statt. Diese Fokusgruppen bzw. Einzelinterviews und deren Auswertungen wurden nach Standards der qualitativen Forschung durchgeführt [14].

Mit den Ergebnissen aus den Fokusgruppen und den Einzelinterviews wurden - in Kombination mit den Ergebnissen der Literatur - insgesamt 17 Fragen zu Motiven und strukturellen Rahmenbedingungen entwickelt. Ergänzt wurden diese Fragen durch Angaben zur Soziodemografie, zu Praxiseigenschaften und zum beruflichen Werdegang. Die Antworten waren mit der Likert-Skala unterlegt. Die 17 Motivationsgründe, die sich aus den Ergebnistabellen ablesen lassen, wurden aus den 3 bereits am Anfang des vorliegenden Methodenabschnitts beschriebenen Perspektiven in Fragen verarbeitet. Die jeweiligen Perspektiven wurden mit einer erklärenden Einleitung eingeführt.

Der erstellte Fragebogen wurde einer Think-Aloud-Testung (3 Sitzungen) mit 9 Teilnehmern, davon 5 Frauen, unterzogen. Die Ergebnisse des Pilottests wurden durch ein multidisziplinäres Team ausgewertet und führten letztlich zur Präzisierung von 4 Items. Nach Abschluss der Fragebogenentwicklung wurde der Erhebungsbogen 20 Ärzten, davon die Hälfte Frauen, die am qualitativen Teil dieser Untersuchung nicht beteiligt waren, zur Prätestung vorgelegt. Als Ergebnis dieser Prätestung mussten 3 Formulierungen wegen sehr heterogenen Interpretationsspielräumen angepasst werden. In einer weiteren Prätestung mit 15 hausärztlichen Teilnehmern, davon 8 Frauen, stellte sich diese Anpassung als trennscharf heraus. Hintergrund war, dass es sich nicht um ein fortlaufendes Erhebungsinstrument handelt und eine Vollerhebung geplant war. Dafür wurde die Validierung mit Cronbachs Alpha durchgeführt [15].

Die Befragung wurde im Versorgungsgebiet der Kassenärztlichen Vereinigung Nordrhein (KVNo) durchgeführt. Der Erhebungszeitraum lag zwischen September 2015 und Februar 2016. Zur Einladung einer Teilnahme und Erläuterung der Erhebung wurde gemeinsam ein Anschreiben entwickelt. Die Wahrung der Anonymität der Teilnehmer wurde durch die postalische Versendung der Fragebögen einschließlich Anschreiben durch die KVNo und die anonyme Rücksendung mittels mitgesendeten frankierten Rückumschlägen an den Schwerpunkt Allgemeinmedizin des Universitätsklinikums Köln gesichert. Die Versendung erfolgte in 2 Wellen. Nach dem Primäranschreiben wurde nach 4 Wochen ein Reminder versendet.

Das Zielkollektiv waren Hausärzte, die sich im Bereich der KVNo in den letzten 5 Jahren neu niedergelassen hatten. Angestellte und ermächtigte Ärzte wurden ausgeschlossen. Als Aufgreifkriterium wurde eine Erstniederlassung im Arztregister der KVNo zwischen 01.01.2009 und 01.05.2015 definiert. Dabei konnte aus datentechnischen Gründen eine frühere Niederlassung außerhalb des genannten KV-Bezirks nicht belastbar ausgeschlossen werden. Die Befragung war als Vollerhebung angesetzt. Die Datensätze der KVNo ergaben zum Zeitpunkt der Erhebung eine Anzahl von 675 hausärztlichen Niederlassungen im genannten Zeitraum.
Die statistische Auswertung erfolgte deskriptiv und induktiv. Mögliche Zusammenhänge zwischen den Gründen für die Niederlassung von Hausärzten und soziodemografischen sowie 4 weiterer Variablen wurden anhand sachlogischer Überlegungen ausfindig gemacht und eine entsprechende Vorselektion potenzieller Einflussfaktoren vorgenommen.

Anschließend wurden signifikante Einflussfaktoren für die jeweiligen Niederlassungsgründe mittels binär-logistischer Regressionsmodelle mit der Zielgröße Niederlassungsgrund „ja“ vs. „nein“ bestimmt. Unabhängige Variablen mit 3 oder mehr kategorialen Ausprägungen wurden in dichotome Variablen transformiert.

Im Detail: Die abhängigen Variablen (Niederlassungsgründe) wurden jeweils dem Fragenblock „Motive und Gründe“ entnommen und dichotomisiert, indem die Antwortkategorien „eher wichtig“ und „wichtig“ respektive „eher unwichtig“ und „unwichtig“ jeweils zu 1 Antwortkategorie zusammengefasst wurden (Niederlassungsgrund ,ja“/,nein“).

Um eine Unterteilung „ländlich/städtisch“ zu ermöglichen, wurde die unabhängige kategoriale Variable Einwohnerzahl dichotomisiert, indem die ursprünglich 5 Antwortkategorien in die beiden Kategorien $<20000$ und $\geq 20000$ Einwohner kondensiert wurden. Die unabhängige kategoriale Variable Alter wurde in die Bereiche $\leq 45$ Jahre und $>45$ Jahre unterteilt, da sich in diesem Bereich im Rahmen einer Voranalyse aufschlussreiche Unterschiede erkennen ließen. Bei der unabhängigen kategorialen Variablen Entscheidungsfindung („Wann haben Sie den Entschluss gefasst, Hausärztin/Hausarzt zu werden?“) wurden die Antwortmöglichkeiten „vor dem Studium“ und „während des Studiums“ aufgrund des beobachteten Verteilungsmusters der Antworten zu 1 Kategorie zusammengefasst und der Antwortmöglichkeit „nach dem Studium“ gegenübergestellt. Schließlich wurde die unabhängige metrische Variable Anzahl Kinder in die binäre Größe „Kinder im Haushalt vorhanden" transformiert, um den Einfluss von Kindern (oder eben keinen) an sich auf die jeweilige Zielgröße untersuchen zu können.

In einem initialen Modell wurden dann jeweils alle vorselektierten Variablen als mögliche Einflussfaktoren berücksichtigt. Anschließend wurden Variablen, die multivariat keinen signifikanten Einfluss zeigten, schrittweise aus dem Gesamtmodell entfernt, um jeweils ein finales Modell mit ausschließlich signifikanten Einflussfaktoren zu erhalten. Angewandt wurde hierbei stets die konditionale Rückwärtsselektion, eine Methode der schrittweisen Variablenauswahl mit einem statistischen Test auf Modell-Aufnahme, der auf der Signifikanz der Score-Statistik beruht, und einem Test auf Ausschluss, der auf der Wahrscheinlichkeit der Likelihood-Quotienten-Statistik basiert.

Abgebildet sind nur optimierte binär-logistische Regressionsmodelle mit unabhängigen Variablen, die einen signifikanten Einfluss auf die Zielvariable haben. Modelle mit weiteren unabhängigen Variablen, die eine schlechtere Performance zeigten, sind nicht dargestellt. Bei den aufgeführten Modellen konnte die Stabilität jeweils per Bootstrapping-Analyse bestätigt werden.

Das Signifikanzniveau wurde auf alpha $=0,05$ festgelegt. Sämtliche statistische Analysen wurden mit der Statistik-Software IBM SPSS 25.0 durchgeführt. 
D Tab. 1 Charakteristiken des Kollektivs (gesamt =437).

\begin{tabular}{|c|c|c|}
\hline & $\begin{array}{l}\text { Angaben } \\
\text { in (\%) }\end{array}$ & $\begin{array}{l}\text { Mittelwert } \\
\text { (min.-max.) } \\
\text { Standard- } \\
\text { abweichung }\end{array}$ \\
\hline \multicolumn{2}{|l|}{ Alterskategorien } & \\
\hline 35 Jahre und jünger & 4,1 & \\
\hline 36-40 Jahre & 25,7 & \\
\hline 41-45 Jahre & 31,0 & \\
\hline 46-50 Jahre & 22,8 & \\
\hline 51-55 Jahre & 11,0 & \\
\hline 56 Jahre und älter & 5,3 & \\
\hline \multicolumn{2}{|l|}{ Geschlecht } & \\
\hline männlich & 49,0 & \\
\hline weiblich & 51,0 & \\
\hline \multicolumn{2}{|l|}{ Familienstand } & \\
\hline alleinlebend & 6,7 & \\
\hline in Partnerschaft/Ehe & 15,9 & \\
\hline in Partnerschaft/Ehe mit Kindern & 73,4 & \\
\hline sonstiges & 3,9 & \\
\hline $\begin{array}{l}\text { Anzahl Kinder im Haushalt } \\
\text { (unabhängig vom Familienstand) }\end{array}$ & & \multirow[t]{6}{*}{$\begin{array}{l}1,69(0-8) \\
\text { SD } 1,19\end{array}$} \\
\hline 0 & 20,8 & \\
\hline 1 & 17,6 & \\
\hline 2 & 38,9 & \\
\hline 3 & 19,0 & \\
\hline mehr als 3 & 3,7 & \\
\hline \multicolumn{2}{|l|}{ Tätigkeit in Praxisform } & \\
\hline Einzelpraxis & 37,7 & \\
\hline BAG-Praxisgemeinschaft & 4,4 & \\
\hline BAG-Gemeinschaftspraxis & 57,9 & \\
\hline \multicolumn{2}{|l|}{ Anzahl Kollegen in Praxis } & \multirow{5}{*}{$\begin{array}{l}1,8(1-8) \\
\text { SD } 1,47\end{array}$} \\
\hline 1 & 68,1 & \\
\hline 2 & 21,5 & \\
\hline 3 & 8,7 & \\
\hline mehr als 3 & 3,9 & \\
\hline \multicolumn{2}{|l|}{ Übernahme eines KV-Sitzes } & \\
\hline Neugründung & 8,4 & \\
\hline Übernahme von Verwandtschaft & 7,7 & \\
\hline Übernahme von befreundeter Person & 7,9 & \\
\hline Übernahme von fremder Person & 76,0 & \\
\hline \multicolumn{2}{|l|}{ Dauer der Niederlassung in Jahren } & \multirow{4}{*}{$\begin{array}{l}3,47(0,25-43) \\
\text { SD } 4,17\end{array}$} \\
\hline bis 2,5 Jahre & 48,0 & \\
\hline über 2,5 bis 5 Jahre & 43,5 & \\
\hline $\begin{array}{l}\text { mehr als } 5 \text { Jahre (frühere } \\
\text { Niederlassung außerhalb der KVNo) }\end{array}$ & 8,5 & \\
\hline
\end{tabular}

- Tab. 1 (Fortsetzung)

\begin{tabular}{|c|c|c|}
\hline & $\begin{array}{l}\text { Angaben } \\
\text { in (\%) }\end{array}$ & $\begin{array}{l}\text { Mittelwert } \\
\text { (min.-max.) } \\
\text { Standard- } \\
\text { abweichung }\end{array}$ \\
\hline \multicolumn{2}{|l|}{ Einwohnerzahl } & \\
\hline bis 20000 & 24,9 & \\
\hline über 20000 bis 100000 & 31,9 & \\
\hline über 100000 & 43,2 & \\
\hline \multicolumn{2}{|c|}{$\begin{array}{l}\text { Entscheidung für hausärztliche } \\
\text { Tätigkeit }\end{array}$} & \\
\hline vor dem Studium & 8,3 & \\
\hline während des Studiums & 13,0 & \\
\hline nach dem Studium & 73,4 & \\
\hline weiß ich nicht mehr & 5,3 & \\
\hline \multicolumn{2}{|l|}{ Approbationserteilung } & \\
\hline bis vor dem Jahr2002 & 50,2 & \\
\hline ab dem Jahr 2002 & 49,8 & \\
\hline
\end{tabular}

\section{Ergebnisse}

Es wurden 675 Fragebögen versendet, von denen insgesamt 446 zurückgeschickt wurden. Nach der ersten Aussendung durch die KVNo waren 373 zurückgesendet worden, was einer Rücklaufquote von 55,3\% entspricht. Nach einem Reminder konnte durch den Rücklauf weiterer 73 Bögen die Quote auf 66,1 \% erhöht werden. Von den insgesamt 446 Fragebögen konnten nach der Plausibilitätsprüfung 437 (64,7\%) ausgewertet werden. Der Prozentsatz fehlender Daten erhöhte sich von < $1 \%$ (Missing) bei den Fragen zum Einfluss auf die Niederlassung auf $5,5 \%$ bei den Fragen zum förderlichen Faktor bis hin zu 6,3\% bei den Fragen zur Erfüllung der Erwartungen. Das Item „externe finanzielle Anreize von Staat, Kommune und Verbände“ weist Missing-Werte von 2,5\%, 16,3\% und 11,7\% auf. Die Testung mit Cronbachs Alpha ergab einen Wert von 0,868. Damit kann von einer hohen Konsistenz und Trennschärfe ausgegangen werden.

Der Großteil (79,5\%) der Befragten war zwischen 36 und 50 Jahre alt. Insgesamt waren 51 \% Frauen im Kollektiv und 73,4\% der Befragten befanden sich im Familienstand Partnerschaft/Ehe mit Kindern. Durchschnittlich befanden sich 1,69 Kinder im Haushalt und 37,7\% hatten sich in einer Einzelpraxis niedergelassen. Die Mehrheit (62,3\%) war in einer Berufsausübungsgemeinschaft mit durchschnittlich 1,8 Kollegen tätig. Von den Teilnehmern der Erhebung waren 43,2\% in einer Großstadt mit mehr als 100000 Einwohnern niedergelassen und 73,4\% hatte sich erst nach dem Studium für eine hausärztliche Tätigkeit entschieden. Die Übernahme eines kassenärztlichen Sitzes erfolgte bei $76 \%$ der Teilnehmer von fremden Vorbesitzern. Von den Befragten waren 16,3\% entsprechend der gezogenen Datensätze zum Zeitpunkt der „aktuellen“ Niederlassung 51 Jahre und älter $(\triangleright$ Tab. 1). 


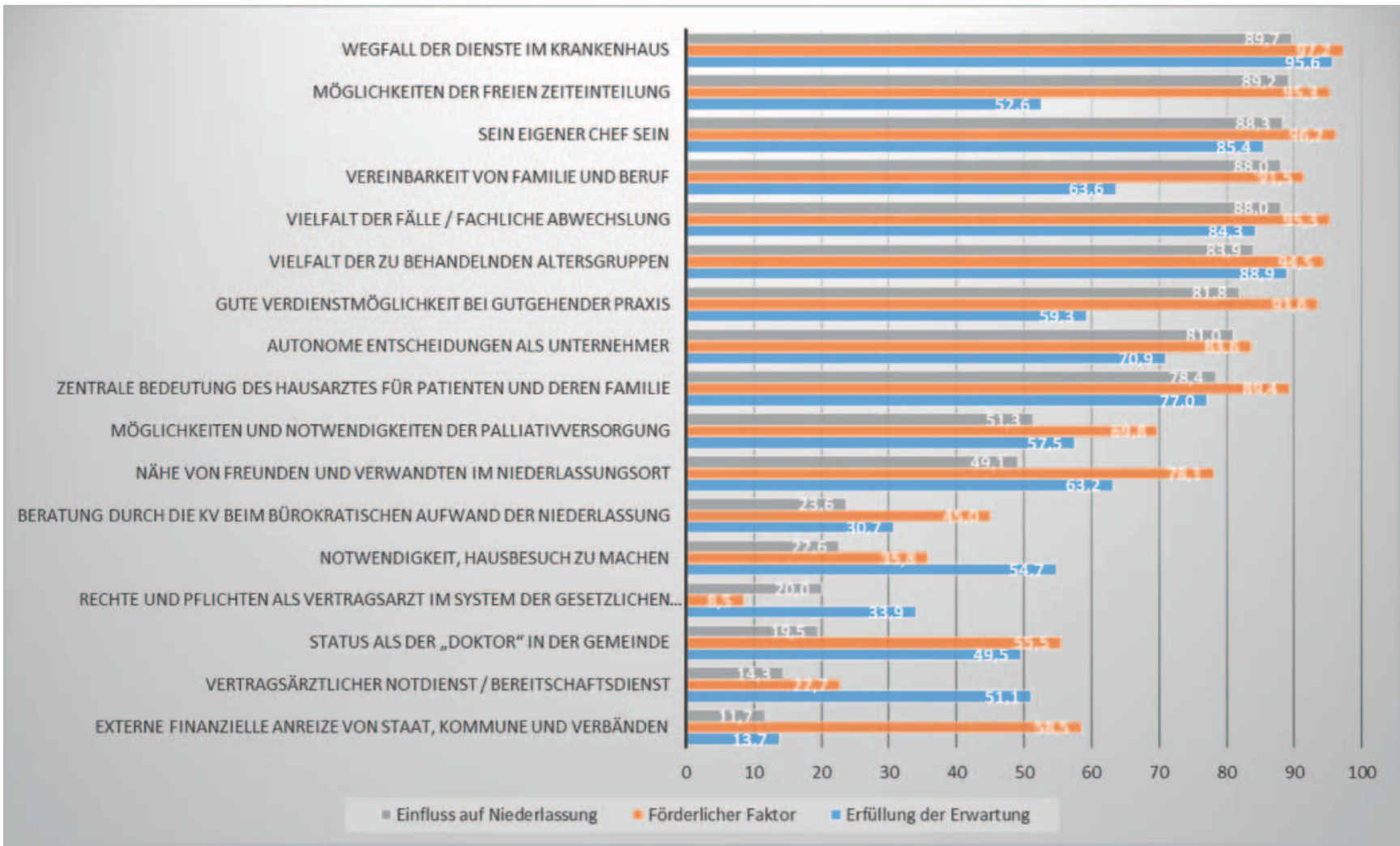

- Abb. 1 Gesamtergebnisse.

In den nachfolgenden Beschreibungen haben sich nach Häufigkeiten der Antwortnennungen 3 Gruppen herauskristallisiert. In der ersten Antwortgruppe besteht eine Zustimmungsrate von mehr als 85 \% bezüglich der untersuchten Faktoren. Bei der zweiten Gruppe liegt die Zustimmungsrate zwischen 44 und $85 \%$ und in der dritten Gruppe liegt sie bei weniger als $44 \%$. Diese Gruppen ziehen sich durch die $\mathbf{A b b} \mathbf{~} \mathbf{1}$ sowie den $>$ Tab. $\mathbf{2}-\mathbf{4}$. Im ersten Frageblock ist die Bedeutung der einzelnen Motive und strukturellen Rahmenbedingungen im Hinblick auf die Niederlassungsentscheidung abgefragt worden. In den vorgenannten Tabellen werden die jeweiligen Häufigkeiten in Prozent angezeigt, die von den Befragten bei den jeweiligen Items auf der 4-stufigen Skala als „wichtig“ oder „eher wichtig“ angekreuzt wurden. Für die - Abb. 1 gilt gleiches in grafischer Form.

Als besonders wichtiger Einfluss auf eine Niederlassung (grüne Balken) wurde das Item „Wegfall der Dienste im Krankenhaus“ $(89,7 \%)$ empfunden. Nach diesem Item folgen noch 4 weitere Items in der Spitzengruppe (> $85 \%$ ). Die „Palliativversorgung“ $(51,4 \%)$ oder „Nähe zu Freunden und Verwandten am Ort der Niederlassung“ (49,1\%) als Vertreter der zweiten Gruppe liegen im Mittelfeld der Relevanz. Die rechtlichen Rahmenbedingungen eines Vertragsarztes finden sich in der letzten Gruppe (<44\%) wieder. Dies sind beispielsweise „Not- bzw. Bereitschaftsdienste“ (14,3\%) oder „Rechte und Pflichten als Vertragsarzt“ (20,0\%), die für eine Niederlassungsentscheidung eher unwichtig waren. In - Abb. 1 sind alle Einzelwerte der Antwortkategorien für den Einfluss auf die Niederlassung (grau), die förderlichen Faktoren (rot) und die erfüllten Erwartungen (blau) dargestellt.
Neben der Relevanz der angebotenen Motive und strukturellen Rahmenbedingungen einer Niederlassung sollten die Befragten aussagen (Mehrfachnennung), welche Faktoren (rote Balken) als förderlich angesehen wurden. Die höchsten Zustimmungswerte erhielten „Wegfall der Dienste im Krankenhaus“ (97,2\%) und „eigener Chef sein“ (96,2\%). In der zweiten Gruppe finden sich „externe finanzielle Anreize durch Staat, Kommune und Verbände“ (58,5\%) und „Beratung durch die KV beim bürokratischen Aufwand der Niederlassung“ (45\%). Die geringsten Zustimmungswerte in der dritten Gruppe erhielten die Items „Rechte und Pflichten als Vertragsarzt“ (8,5\%) und - spiegelbildlich zum Motiv „Wegfall der Krankenhausdienste“ - der „vertragsärztliche Notdienst/Bereitschaftsdienst“ (22,7\%).

Abschließend sollte eingeschätzt werden, ob die Erwartungen (blaue Balken) erfüllt wurden. Der „Wegfall der Dienste“ (95,6\%) und die „Vielfalt der zu behandelnden Altersgruppen“ (88,9\%) wurden am ehesten erfüllt. Die Erwartung an „Status als der Doktor in der Gemeinde“ (49,5\%) wurde durchschnittlich erfüllt. Dagegen werden mit 13,7\% Zustimmung „externe finanzielle Anreize von Staat, Kommune und Verbänden“ als am wenigsten erfüllt angesehen.

Betrachtet man das Antwortverhalten auf geschlechts-, altersoder praxisspezifische Unterschiede, so ist festzustellen, dass bei männlichen Teilnehmern die Erlangung von Autonomie in der unternehmerischen Entscheidungsfindung gegenüber den weiblichen Teilnehmern als eine signifikant deutlichere Motivation zur Niederlassung gilt, als ein signifikant förderlicherer Faktor und als signifikant erfülltere Erwartung zu sehen ist. 
- Tab. 2 Ergebnisse $(n=437)$ nach Geschlechtern (altersunabhängig).

\begin{tabular}{|c|c|c|c|c|c|c|}
\hline \multirow[t]{2}{*}{ Item } & \multicolumn{2}{|c|}{ Einfluss auf Niederlassung (\%) } & \multicolumn{2}{|c|}{ Förderlicher Faktor (\%) } & \multicolumn{2}{|c|}{ Erfüllung d. Erwartung (\%) } \\
\hline & $\begin{array}{l}w \\
n=222\end{array}$ & $\begin{array}{l}m \\
n=215\end{array}$ & $\begin{array}{l}w \\
n=222\end{array}$ & $\begin{array}{l}m \\
n=215\end{array}$ & $\begin{array}{l}w \\
n=222\end{array}$ & $\begin{array}{l}m \\
n=215\end{array}$ \\
\hline autonome Entscheidungen als Unternehmer & 76,0 & 86,7 & 78,2 & 88,9 & 65,6 & 76,7 \\
\hline sein eigener Chef sein & 85,2 & 91,5 & 96,3 & 96,2 & 83,1 & 87,7 \\
\hline $\begin{array}{l}\text { Rechte und Pflichten als Vertragsarzt im System } \\
\text { der gesetzlichen Krankenversicherung }\end{array}$ & 18,7 & 20,5 & 8,4 & 8,3 & 31,6 & 36,6 \\
\hline $\begin{array}{l}\text { Beratung durch die KV beim bürokratischen } \\
\text { Aufwand der Niederlassung }\end{array}$ & 26,7 & 19,6 & 46,2 & 43,3 & 29,0 & 32,3 \\
\hline Vielfalt der Fälle/fachliche Abwechslung & 91,4 & 84,2 & 96,8 & 93,8 & 87,1 & 80,7 \\
\hline Vielfalt der zu behandelnden Altersgruppen & 89,6 & 77,8 & 96,3 & 93,3 & 92,5 & 85,1 \\
\hline $\begin{array}{l}\text { Möglichkeiten und Notwendigkeiten der } \\
\text { Palliativversorgung }\end{array}$ & 56,8 & 45,5 & 64,1 & 65,5 & 63,1 & 51,7 \\
\hline Vereinbarkeit von Familie und Beruf & 89,6 & 86,3 & 93,5 & 89,4 & 66,7 & 59,4 \\
\hline Möglichkeiten der freien Zeiteinteilung & 92,8 & 85,2 & 96,8 & 93,7 & 56,6 & 46,5 \\
\hline Wegfall der Dienste im Krankenhaus & 92,3 & 86,8 & 97,2 & 97,1 & 97,6 & 93,5 \\
\hline vertragsärztlicher Notdienst/Bereitschaftsdienst & 16,9 & 11,3 & 20,5 & 24,1 & 53,4 & 49,2 \\
\hline $\begin{array}{l}\text { gute Verdienstmöglichkeit bei gutgehender } \\
\text { Praxis }\end{array}$ & 73,4 & 84,3 & 94,8 & 92,2 & 52,5 & 66,5 \\
\hline $\begin{array}{l}\text { externe finanzielle Anreize von Staat, Kommune } \\
\text { und Verbänden }\end{array}$ & 11,9 & 11,2 & 59,0 & 57,5 & 14,0 & 13,6 \\
\hline $\begin{array}{l}\text { zentrale Bedeutung des Hausarztes für Patienten } \\
\text { und deren Familie }\end{array}$ & 80,6 & 75,9 & 90,4 & 88,3 & 82,5 & 70,9 \\
\hline Status als der „Doktor“ in der Gemeinde & 18,1 & 21,2 & 55,5 & 55,0 & 57,0 & 41,6 \\
\hline Notwendigkeit, Hausbesuche zu machen & 18,6 & 26,6 & 36,1 & 35,4 & 54,8 & 54,6 \\
\hline $\begin{array}{l}\text { Nähe von Freunden und Verwandten im } \\
\text { Niederlassungsort }\end{array}$ & 50,9 & 46,7 & 79,6 & 76,3 & 65,0 & 60,8 \\
\hline
\end{tabular}

Bei befragten Hausärztinnen bestand gegenüber den Hausärzten eine höhere Motivation zur Niederlassung bezogen auf fachliche Vielfalt der Fälle, freie Zeiteinteilung und Wegfall der Krankenhausdienste. Betrachtet man die Erwartungserfüllung, so sind neben der fachlichen Vielfalt der Fälle und Vielfalt der Altersgruppen auch bei der Möglichkeit der Palliativversorgung bei den weiblichen Befragten signifikant mehr die Erwartungen erfüllt worden als bei männlichen Befragten.

In der Betrachtung der Altersgruppen zeigt sich, dass als Motivation zur Niederlassung die „Autonomie“ und „eigener Chef sein“ bei den unter 45-Jährigen signifikant häufiger genannt wurde. Dies gilt auch für „Vereinbarkeit von Familie und Beruf“. Bei der Nennung der förderlichen Faktoren wurde die „Beratung durch die KV“ von den über 45-Jährigen signifikant häufiger genannt. Bei den erfüllten Erwartungen wurde bei den unter 45-Jährigen signifikant häufiger „Verdienstmöglichkeiten“ und bei den über 45-Jährigen die „Notwendigkeit der Hausbesuche“ genannt.

Die Ergebnisse der binären Regressionsanalyse haben, außer für den „Familienstand“ (kein Prädiktor), die in $>$ Tab. 4 aufge- führten Prädiktoren hervorgebracht. Dargestellt werden hier nur die Prädiktoren, die einen signifikanten Wert erzielt haben. Der häufigste Prädiktor ist das „Geschlecht“. Frauen werden eher durch die „Vereinbarkeit von Familien und Beruf“ (OR 2,106; $95 \%-K I$ 1,349-3,287; $\mathrm{p}=0,001)$ und „freie Zeiteinteilung“ (OR 1,611; 95 \%-KI 1,069-2,429; p=0,023) zur Niederlassung motiviert. In diesem Kontext gliedert sich vor allem der „Wegfall der Dienste“ ein (OR 1,590; $95 \%-K I$ 1,058-2,389; $\mathrm{p}=0,026$ ). Zusätzlich motiviert wird die Niederlassung in der Form, dass der Ort der Niederlassung in der „Nähe von Freunden und Verwandten“ (OR 1,643; 95 \%-KI 1,013-2,664; $p=0,044)$ liegt. Bei Männern dagegen spielen die „Selbständigkeit“ (OR 0,653; $95 \%$-KI 0,434-0,982; $p=0,041$ ) bzw. „Entscheidungsfreiheit“ (OR 0,613; $95 \%$-KI 0,408-0,923; $p=0,019$ ) eine wichtige Rolle in der Niederlassungsmotivation. Gleiches gilt auch für die „Verdienstmöglichkeiten“ (OR 0,507; $95 \%-K I$ 0,315-0,816; p=0,005).

Die Wahl der Praxisform (Einzel- vs. Berufsausübungsgemeinschaft (BAG)) ist ein gleichhäufiger Prädiktor wie das Alter ( $\leq 45 \mathrm{~L}$ ] vs. > 45 LJ). Die „üngeren“ motiviert eine Niederlassung wegen 
- Tab. 3 Ergebnisse ( $(=437)$ nach Altersgruppen (geschlechtsunabhängig).

\begin{tabular}{|c|c|c|c|c|c|c|}
\hline \multirow[t]{2}{*}{ Item } & \multicolumn{2}{|c|}{ Einfluss auf Niederlassung (\%) } & \multicolumn{2}{|c|}{ Förderlicher Faktor (\%) } & \multicolumn{2}{|c|}{ Erfüllung d. Erwartung (\%) } \\
\hline & $\begin{array}{l}\leq 45 . \text { L U } \\
n=265\end{array}$ & $\begin{array}{l}>45 . \text { L J } \\
n=170\end{array}$ & $\begin{array}{l}\leq 45 . \text { LU } \\
n=265\end{array}$ & $\begin{array}{l}>45 . \text { L J } \\
n=170\end{array}$ & $\begin{array}{l}\leq 45 . \text { Lᄂ } \\
n=265\end{array}$ & $\begin{array}{l}>45 . \text { L J } \\
n=170\end{array}$ \\
\hline autonome Entscheidungen als Unternehmer & 82,2 & 78,3 & 85,1 & 80,9 & 75,7 & 63,9 \\
\hline sein eigener Chef sein & 91,4 & 86,4 & 97,3 & 94,4 & 88,5 & 81,1 \\
\hline $\begin{array}{l}\text { Rechte und Pflichten als Vertragsarzt im System } \\
\text { der gesetzlichen Krankenversicherung }\end{array}$ & 16,4 & 24,7 & 8,5 & 8,8 & 33,4 & 35,0 \\
\hline $\begin{array}{l}\text { Beratung durch die KV beim bürokratischen } \\
\text { Aufwand der Niederlassung }\end{array}$ & 21,4 & 25,7 & 40,3 & 52,5 & 27,4 & 36,1 \\
\hline Vielfalt der Fälle/fachliche Abwechslung & 87,0 & 89,3 & 95,1 & 95,7 & 84,0 & 84,7 \\
\hline Vielfalt der zu behandelnden Altersgruppen & 83,1 & 85,2 & 94,6 & 95,1 & 88,3 & 90,3 \\
\hline $\begin{array}{l}\text { Möglichkeiten und Notwendigkeiten der } \\
\text { Palliativversorgung }\end{array}$ & 47,3 & 57,4 & 68,5 & 72,7 & 56,0 & 60,0 \\
\hline Vereinbarkeit von Familie und Beruf & 90,5 & 84,6 & 92,4 & 90,1 & 65,5 & 60,0 \\
\hline Möglichkeiten der freien Zeiteinteilung & 91,0 & 86,3 & 95,8 & 94,5 & 53,4 & 51,5 \\
\hline Wegfall der Dienste im Krankenhaus & 91,3 & 86,9 & 97,7 & 96,3 & 95,1 & 96,3 \\
\hline vertragsärztlicher Notdienst/Bereitschaftsdienst & 11,0 & 19,8 & 24,2 & 19,9 & 48,4 & 55,3 \\
\hline $\begin{array}{l}\text { gute Verdienstmöglichkeit bei gutgehender } \\
\text { Praxis }\end{array}$ & 82,6 & 80,5 & 93,8 & 93,8 & 64,3 & 52,5 \\
\hline $\begin{array}{l}\text { externe finanzielle Anreize von Staat, Kommune } \\
\text { und Verbänden }\end{array}$ & 8,9 & 15,7 & 55,7 & 63,2 & 15,1 & 11,8 \\
\hline $\begin{array}{l}\text { zentrale Bedeutung des Hausarztes für Patienten } \\
\text { und deren Familie }\end{array}$ & 79,8 & 78,1 & 89,8 & 89,3 & 76,3 & 78,4 \\
\hline Status als der „Doktor“ in der Gemeinde & 21,1 & 17,2 & 58,9 & 50,0 & 49,7 & 49,0 \\
\hline Notwendigkeit, Hausbesuch zu machen & 20,0 & 26,4 & 35,5 & 36,1 & 53,7 & 56,3 \\
\hline $\begin{array}{l}\text { Nähe von Freunden und Verwandten im } \\
\text { Niederlassungsort }\end{array}$ & 46,8 & 52,7 & 78,3 & 79,0 & 61,9 & 65,2 \\
\hline
\end{tabular}

„Entscheidungsfreiheit“ (OR 0,509; $95 \%$ KI 0,329-0,789; $\mathrm{p}=0,002)$, „Selbstständigkeit“ (OR 0,628; $95 \%-K I ; 0,414-0,952$; $\mathrm{p}=0,028$ ), „Wegfall der Krankenhausdienste“ (OR 0,523; $95 \%-\mathrm{KI}$ $0,344-0,794 ; p=0,002$ ), „freie Zeiteinteilung“ (OR 0,527; 95\%-KI $0,345-0,803 ; p=0,003$ ) und „Vereinbarkeit von Familie und Beruf“ (OR 0,462; $95 \%-K I$ 0,293-0,727; p=0,001). Die Praxisform BAG korreliert positiv mit dem Motivationsfaktor „Vereinbarkeit von Familien und Beruf" (OR 2,148; 95\%-KI 0,1380-3,344; p=0,001). Ist die Einzelpraxis die Wahl der Niederlassungswilligen, sind diese besonders durch „Entscheidungsfreiheit“ (OR 0,597; 95 \%-KI 0,389-0,918; $p=0,019$ ) motiviert. Aber auch die „Möglichkeit der KV-Not-/Bereitschaftsdienste“ (OR 0,283; $95 \%$-KI 0,095-0,845; $p=0,024)$ scheint für die Einzelpraxis-Wahl ein Stimulator zu sein.

Wie bereits bei den Prädiktoren Geschlecht und Praxisform spielt der Prädiktor Kinder im Haushalt (nein vs. ja) bei „Vereinbarkeit von Familie und Beruf“ bzw. „freie Zeiteinteilung“ eine wichtige Rolle, wenn eine Niederlassungsmotivation besteht. Umgekehrt wird ohne Kinder im Haushalt die Niederlassungsmotivation durch „KV-Beratung“ getrieben.
Auch die räumliche Lage (Einwohnerzahl $\leq 20000$ vs. > 20000 ) gilt als Prädiktor. In ländlichen Regionen wird die Niederlassungsmotivation durch „externe finanzielle Anreize“ (hier Anreize durch die Gemeinde) (OR 0,166; 95\%-KI 0,036-0,766; $p=0,021$ ) oder durch die „Krankheitsvielfalt“ getriggert (OR 0,641; $95 \%$-KI 0,432-0,951; $p=0,027$ ).

Lag der Entscheidungszeitpunkt (vor bzw. während Studium vs. später) für die Fachrichtung Allgemeinmedizin und damit hausärztliche Tätigkeit schon vor oder während des Studiums, sind die „zentrale Bedeutung des Hausarztes“ bzw. das „Statussymbol Hausarzt“ eher eine Niederlassungsmotivation. Umgekehrt stellt nach dem Studium der „Wegfall der Dienste“ eine Motivation zur Niederlassung dar (OR 2,594; 95 \%-KI 1,290-5,213; $p=0,007)$.

Für die Items (nicht in Tabelle enthalten) „Rechte und Pflichten als Vertragsarzt“, „Notwendigkeit von Hausbesuchen“, „Chance der Palliativversorgung“ und „Vielfalt der Altersgruppen“ ergab sich für keinen der aufgeführten Prädiktoren ein signifikanter Einfluss. 
- Tab.4 Signifikante Prädiktoren der binär-logistischen Regressionsanalyse ${ }^{1}$.

\begin{tabular}{|c|c|c|c|c|c|c|c|c|c|c|c|c|c|c|c|c|c|c|c|}
\hline \multicolumn{2}{|c|}{ Unabhängige Variablen } & \multicolumn{3}{|c|}{$\begin{array}{l}\text { Alter } \\
\text { ( } \leq 45 \text { LJ vs. }>45 \text { LJ) }\end{array}$} & \multicolumn{3}{|c|}{$\begin{array}{l}\text { Geschlecht } \\
\text { (männlich vs. weiblich) }\end{array}$} & \multicolumn{3}{|c|}{$\begin{array}{l}\text { Praxisform } \\
\text { (Einzelpraxis vs. BAG) }\end{array}$} & \multicolumn{3}{|c|}{$\begin{array}{l}\text { Einwohnerzahl } \\
(\leq 20000 \text { vs. }>20000)\end{array}$} & \multicolumn{3}{|c|}{$\begin{array}{l}\text { Kinder im Haushalt } \\
\text { (kein Kind vs. mind. } \\
1 \text { Kind) }\end{array}$} & \multicolumn{3}{|c|}{$\begin{array}{l}\text { Entscheidungsfindung } \\
\text { (vor/währ. vs. nach Stud.) }\end{array}$} \\
\hline $\begin{array}{l}\text { abhängige } \\
\text { Variablen }\end{array}$ & $\mathrm{R}^{2}$ Nagelkerke & OR & $95 \%-K I$ & $\mathrm{P}$ & $\mathrm{OR}$ & $95 \%-K I$ & $\mathrm{P}$ & $\mathrm{OR}$ & $95 \%-K I$ & $\mathrm{P}$ & OR & $95 \%-K I$ & $\mathrm{P}$ & $\mathrm{OR}$ & $95 \%-K I$ & $\mathrm{P}$ & $\mathrm{OR}$ & $95 \%-K I$ & $\mathrm{P}$ \\
\hline $\begin{array}{l}\text { Entschei- } \\
\text { dungsfrei- } \\
\text { heit }\end{array}$ & 0,074 & 0,509 & $\begin{array}{l}0,329- \\
0,789\end{array}$ & 0,002 & 0,613 & $\begin{array}{l}0,408- \\
0,923\end{array}$ & 0,019 & 0,597 & $\begin{array}{l}0,389- \\
0,918\end{array}$ & 0,019 & & & & & & & & & \\
\hline $\begin{array}{l}\text { Selbststän- } \\
\text { digkeit }\end{array}$ & 0,036 & 0,628 & $\begin{array}{l}0,414- \\
0,952\end{array}$ & 0,028 & 0,653 & $\begin{array}{l}0,434- \\
0,982\end{array}$ & 0,041 & & & & & & & & & & & & \\
\hline $\begin{array}{l}\text { KV-Bera- } \\
\text { tung }\end{array}$ & 0,059 & & & & & & & & & & & & & 0,341 & $\begin{array}{l}0,134- \\
0,872\end{array}$ & 0,025 & & & \\
\hline $\begin{array}{l}\text { Krankheits- } \\
\text { vielfalt }\end{array}$ & 0,067 & & & & & & & & & & 0,641 & $\begin{array}{l}0,432- \\
0,951\end{array}$ & 0,027 & & & & & & \\
\hline $\begin{array}{l}\text { Vereinbar- } \\
\text { keit mit } \\
\text { Familie }\end{array}$ & 0,177 & 0,462 & $\begin{array}{l}0,293- \\
0,727\end{array}$ & 0,001 & 2,106 & $\begin{array}{l}1,349- \\
3,287\end{array}$ & 0,001 & 2,148 & $\begin{array}{l}0,1380- \\
3,344\end{array}$ & 0,001 & & & & 3,355 & $\begin{array}{l}1,948- \\
5,779\end{array}$ & $<0,001$ & & & \\
\hline $\begin{array}{l}\text { freie Zeit- } \\
\text { einteilung }\end{array}$ & 0,054 & 0,527 & $\begin{array}{l}0,345- \\
0,803\end{array}$ & 0,003 & 1,611 & $\begin{array}{l}1,069- \\
2,429\end{array}$ & 0,023 & & & & & & & 1,682 & $\begin{array}{l}1,011- \\
2,800\end{array}$ & 0,045 & & & \\
\hline $\begin{array}{l}\text { Wegfall der } \\
\text { KH-Dienste }\end{array}$ & 0,091 & 0,493 & $\begin{array}{l}0,243- \\
0,1000\end{array}$ & 0,049 & 3,021 & $\begin{array}{l}1,416- \\
6,445\end{array}$ & 0,004 & & & & & & & & & & 2,594 & $\begin{array}{l}1,290- \\
5,213\end{array}$ & 0,007 \\
\hline $\begin{array}{l}\text { KV-Not- } \\
\text { dienst }\end{array}$ & 0,106 & & & & & & & 0,283 & $\begin{array}{l}0,095- \\
0,845\end{array}$ & 0,024 & & & & & & & & & \\
\hline $\begin{array}{l}\text { Verdienst- } \\
\text { möglichkeit }\end{array}$ & 0,028 & & & & 0,507 & $\begin{array}{l}0,315- \\
0,816\end{array}$ & 0,005 & & & & & & & & & & & & \\
\hline $\begin{array}{l}\text { externe fi- } \\
\text { nanzielle } \\
\text { Anreize }\end{array}$ & 0,076 & & & & & & & & & & 0,166 & $\begin{array}{l}0,036- \\
0,766\end{array}$ & 0,021 & & & & & & \\
\hline $\begin{array}{l}\text { Bedeutung } \\
\text { der Haus- } \\
\text { arztrolle }\end{array}$ & 0,046 & & & & & & & & & & & & & & & & 0,485 & $\begin{array}{l}0,305- \\
0,770\end{array}$ & 0,002 \\
\hline $\begin{array}{l}\text { Statussym- } \\
\text { bol Hausarzt }\end{array}$ & 0,097 & & & & & & & & & & & & & & & & 0,249 & $\begin{array}{l}0,092- \\
0,676\end{array}$ & 0,006 \\
\hline $\begin{array}{l}\text { Freunde und } \\
\text { Verwandte }\end{array}$ & 0,015 & & & & 1,643 & $\begin{array}{l}1,013- \\
2,664\end{array}$ & 0,044 & & & & & & & & & & & & \\
\hline
\end{tabular}

1 dargestellt sind jeweils die im finalen Modell enthaltenen unabhängigen Variablen (1. Zeile) und die Motivationsgründe zur Niederlassung (1. Spalte) jeweils als abhängige Variablen. 


\section{Diskussion}

Die vorliegende Untersuchung sollte ermitteln, welche Motive und strukturellen Rahmenbedingungen die Entscheidung für die Niederlassung beeinflussen, welche Faktoren einen förderlichen Aspekt aufweisen und ob sich die Erwartungen an die Niederlassung erfüllt haben. Bisherige Studien konzentrierten sich vorrangig auf Medizinstudierende oder Ärzte in Weiterbildung [8, 1620]. In der vorliegenden Arbeit handelt es sich um eine Vollerhebung in der drittgrößten KV Deutschlands gemessen an der Anzahl ihrer Mitglieder. Die hohe Responserate kann als Indikator für ein großes Interesse in der Hausärzteschaft am Thema Nachwuchsförderung gewertet werden. Zudem lässt sich an der Kategorie „Erfüllung“ ablesen, dass die Befragten ihre hausärztliche Tätigkeit als erfüllende Arbeit wahrnehmen. Denn im biografischen Teil der Studie beantworteten $97 \%$ der Teilnehmer die Frage, ob sie eine Niederlassung wiederholen würden, mit „ja“.

Im Gegensatz zu bisherigen Befragungen zeigt diese Untersuchung von Hausärzten nach „aktueller“ Niederlassung, dass die meisten Befragten 41 Jahre und älter waren. Bisherige Förderprogramme haben diese Altersgruppe wenig bis gar nicht berücksichtigt. Es werden vorwiegend Studenten beworben, die noch keine Fachrichtung ausgewählt haben, bzw. Weiterbildungsassistenten, deren Lebensplanung in einer sich noch entwickelnden Phase, wie beispielweise Familienplanung, steckt [19]. In der vorliegenden Untersuchung ist festzustellen, dass $75 \%$ der Befragten erst (lange) nach dem Studium eine Entscheidung für eine hausärztliche Tätigkeit getroffen haben. Bei der Betrachtung nach Geschlechtern zeigen sich geringfügige spezifische Unterschiede. Bei dem Vergleich der Altersgruppen kann festgestellt werden, dass eine Niederlassung in ländlichen Regionen ab dem 46. Lebensjahr deutlich zunimmt. Umkehrschluss könnte sein, dass eine Niederlassung der Jüngeren möglicherweise mit den geänderten Lebensformen einhergehen könnte [21]. In anderen Untersuchungen wurde festgestellt, dass Ärzte, die auf dem Land aufgewachsen sind, sich dort eher niederlassen als Städter $[19,22,23]$.

Aus den Ergebnissen der Befragung ergeben sich als Vorteile einer Niederlassung „weitgehende berufliche Autonomie“, „abwechslungsreiche Tätigkeit“ und „hohes Einkommen“. Diese 3 Faktoren können aufgrund der hohen Prozentzahlen als Pull-Faktoren (Schaffung von Vorteilen) gelten. Eine hohe Bewertung hat auch das Motiv „Wegfall der Dienste“ erhalten. Allerdings ist dieses Motiv, da Dienste als Nachteile eines Beschäftigungsverhältnisses gesehen werden, als Push-Faktor (Abbau von Nachteilen) zu werten. Diesen Faktoren mit hoher Wichtigkeit stehen Antworten wie „Bürokratie und rechtlicher Rahmen“, „Teilnahme am Bereitschaftsdienst“ und „Notwendigkeit von Hausbesuchen“ mit geringen Werten gegenüber. Diese sind als abschreckend zu bewerten.

Betrachtet man die Motive untereinander, dann schneiden diejenigen am schlechtesten ab, die für eine Niederlassung vom Gesundheitssystem gesteuert sind, wie beispielsweise „Notwendigkeit der Hausbesuche“ oder „Not- bzw. Bereitschaftsdienste“. Deren Wichtigkeitsbeurteilungen liegen knapp über, meistens aber unter $20 \%$ für eine Niederlassungsmotivation. Vor allem von Seiten der KVNo scheint es anderweitige Anstrengungen geben zu müssen, um mehr Antrieb für eine Niederlassung zu schaffen. Zwar wurde die Beratung durch die KVNo mit ca. $45 \%$ als förderlich wahrgenommen, aber als Antrieb und im Hinblick auf die Erfüllung der Erwartungen bleibt die Wirklichkeit dahinter zurück. Es scheint also, dass die Beratung keine Wichtigkeit bei der Motivation zur Niederlassung besitzt. Interessanter ist im Hinblick auf die Niederlassungsmotivation die Wichtigkeit „Nähe zu Freunden und Verwandten am Niederlassungsort“. Hier zeichnet sich ein „Klebeeffekt“ ab. Der lässt sich in Bezug auf die Niederlassungsmotivation nicht durch extern beeinflussen. Allerdings könnte diese Erkenntnis helfen, Zielgruppen besser zu erkennen und anzusprechen.

Bei der Ergebnisdurchsicht zur Erwartung kann abgelesen werden, dass die meisten Befragten ihre Erwartungen erfüllt sehen. Die Frage nach der Erfüllung der Erwartungen relativiert bei einigen förderlichen oder abschreckenden Motiven das Ausmaß. Zusammenfassend kann z. B. gesagt werden, dass „Work-Life-Balance“-Motive oder Verdienstmöglichkeiten erfüllt wurden, aber nicht in dem Maß, wie es als förderlicher Faktor gesehen wurde. Andersherum wurden die Bereitschaftsdienste bzw. Hausbesuche, die als abschreckend bewertet wurden, im Nachhinein als weniger schrecklich wahrgenommen. Offensichtlich hat sich die Mehrheit damit arrangiert.

Die Gewinnung von (haus-) ärztlichem Nachwuchs ist derzeit in Stereotypen gefangen [24]. Vielfach werden entsprechend $\S 105$ SGB V monetäre Anreize gesetzt. Hintergrund ist der § 99 SGB V, der zwar die Überversorgung, nicht aber die Unterversorgung bekämpft. Andere Anreize wie beispielsweise Angebote zur Kinderbetreuung oder Schaffung von Arbeitsplätzen für den Lebenspartner am potenziellen Niederlassungsort sind von Seiten der KV nicht zu leisten. Da sind andere politische Akteure in der Verpflichtung [25-28].

Die Ergebnisse legen die Vermutung nahe, dass die „Pull-Faktoren“ wie weitgehende berufliche Autonomie, abwechslungsreiche Tätigkeit oder hohes Einkommen noch stärker bei potenziell niederlassungswilligen Ärztinnen und Ärzten kommuniziert werden müssen. Zudem scheint es von großer Bedeutung zu sein, dass die „Push-Faktoren“ wie Bürokratie und rechtlicher Rahmen, Teilnahme am Bereitschaftsdienst oder die Notwendigkeit von Hausbesuchen von der Selbstverwaltung reformiert werden sollten. Zudem lassen die demografischen Angaben der Befragten den Schluss zu, dass die Förderstrategien stärker in Richtung der bereits im Gesundheitssystem etablierten Ärzte ausgerichtet werden sollten [29-31].

\section{Stärken und Schwächen}

Die Studie weist ein repräsentatives Meinungsbild niedergelassener Hausärztinnen und -ärzte in der Kassenärztlichen Vereinigung Nordrhein auf. Bei allen Antworten sei auf die von der regionalen KV vorgegebenen Niederlassungsmöglichkeiten/-bedingungen verwiesen. Anders als Befragungen von Studierenden oder Medizinern in der Weiterbildung, die prospektiv Wünsche an das zukünftige Berufsprofil dokumentieren, konnte das hier befragte Kollektiv auf eigene Erfahrungen zurückgreifen und die Motive und strukturellen Rahmenbedingungen einem „Realitäts-Check“ 
unterziehen. Im Hinblick auf die Aussagen nach den Motiven der Niederlassung ist darauf hinzuweisen, dass nicht nach „Zustimmung“ oder „Ablehnung“ gefragt wurde, sondern nach persönlicher „Wichtigkeit“. Damit kann von einem hohen Individualitätsfaktor im Antwortverhalten ausgegangen werden.

Eine klassische Validierung des Fragebogens konnte nicht vorgenommen werden, da eine anschließende Vollerhebung nicht mehr möglich gewesen wäre. Eine weitere Limitation ist die Fragenauswahl bzw. -formulierung. Im Hinblick auf die 3 Kategorien Motivation, förderlicher Faktor und Erwartungserfüllung sind die Fragen in Kompatibilitätsform erstellt worden. Dies schränkt die Interpretationsmöglichkeiten ein, lässt aber Trends ablesen. Trotz weiterer möglicher Faktoren, die sich aus der Literatur bzw. den Fokusgruppen ergeben haben, wurde die Anzahl gezielt begrenzt. Erfahrungsgemäß sinkt die Rücklaufquote mit steigender Anzahl der Fragen.

\section{Schlussfolgerungen}

Aus Sicht der Autoren erscheinen die derzeitigen Förderprogramme zur Niederlassung als Hausärztin bzw. Hausarzt als zu pauschal. Eine gezielte Bewerbung der unterschiedlichsten Gruppen entsprechend der Prädiktoren unter Verstärkung der genannten Pull-Faktoren sollte angestrebt werden. Darüber hinaus sollten die politischen Entscheider versuchen, die Fehlinformationen über die Belastungen im Notdienst oder die verringerten Verdienstmöglichkeiten zu entkräften. Eine Überarbeitung der derzeitigen Strategie scheint geboten.

\section{KERNAUSSAGEN:}

- Die derzeitige Motivation zur Niederlassung liegt in der Flucht vor den Nachteilen im stationären Sektor.

- Die Strategie zur Motivation einer Niederlassung ist zu wenig zielgruppenorientiert.

- Nicht die Push-Faktoren (Vermeidung von Nachteilen durch Veränderung), sondern die Pull-Faktoren (Erzielung von Vorteilen durch Veränderung) können die Niederlassung attraktiver machen.

\section{Interessenkonflikt}

Miguel Tamayo erklärt, dass er an Entscheidungen über Fördermaßnahmen der Kassenärztlichen Vereinigung nach §105 Abs. 1a SGB V mitwirkt. Alle weiteren Autoren weisen keinen Interessenkonflikt auf.

\section{Literatur}

[1] Kopetsch T. Dem deutschen Gesundheitswesen gehen die Ärzte aus! - Studie zur Altersstruktur und Arztzahlentwicklung. Berlin: Bundesärztekammer, Kassenärztliche Vereinigung. 2010. Im Internet (Stand: 20.04.2019): 5. Aufl: http://www.kbv.de/media/sp/Arztzahlstudie_2010.pdf

[2] Kaduszkiewicz H, Teichert U, van den Busche H. Ärztemangel in der hausärztlichen Versorgung auf dem Lande und im Öffentlichen Gesund- heitsdienst. Bundesgesundheitsblatt Gesundheitsforschung Gesundheitsschutz 2018; 61: 187-194

[3] Deutscher Ärzteverlag. Landarzt gesucht: Förderprogramme für den medizinischen nachwuchs (01.02.2018). Im Internet (Stand: 29.07.2018): https://www.operation-karriere.de/karriereweg/medizin studium/landarzt-gesucht-foerderprogramme-fuer-den-medizinischennachwuchs.html

[4] Kalitzkus V, Abholz HH. Letzter Landarzt oder Arzt der Zukunft? Z Allg Med 2017; 93: 109-112

[5] Bärnighausen T, Bloom DE. Financial Incentives for return of services in underserved areas: systematic review (May 2008). Im Internet (Stand: 12.04.2019): https://cdn1.sph.harvard.edu/wp-content/uploads/sites/ 1288/2013/10/PGDA_WP_36.pdf

[6] Djalali S, Frei A, Rosemann T et al. Weiterbildung zum Hausarzt - ein europäischer Vergleich. Praxis (Bern) 2013; 102: 327-333

[7] Blozik E, Ehrhardt M, Scherer M. Förderung des allgemeinmedizinischen Nachwuchses. Initiativen in der universitären Ausbildung von Medizinstudierenden. Bundesgesundheitsblatt Gesundheitsforschung Gesundheitsschutz 2014; 57: 892-902

[8] Schneider A, Karsch-Volk M, Rupp A et al. Determinanten für eine hausärztliche Berufswahl unter Studierenden der Medizin: Eine Umfrage an drei bayerischen Medizinischen Fakultäten. GMS Z Med Ausbild 2013; 30: doi:10.3205/zma000888

[9] Roick C, Heider D, Gunther $\mathrm{OH}$ et al. Was ist künftigen Hausärzten bei der Niederlassungsentscheidung wichtig? Ergebnisse einer postalischen Befragung junger Ärzte in Deutschland. Gesundheitswesen 2012; 74: $12-20$

[10] Behmann M, Schmiemann G, Lingner H. Berufszufriedenheit von Hausärzten-Ergebnisse einer Befragung. Dtsch Arztebl Int 2012; 109: 193200

[11] Steinhaeuser J, Joos S, Szecsenyi J et al. A comparison of the workload of rural and urban primary care physicians in Germany: analysis of a questionnaire survey. BMC Fam Pract 2011; 12: 112

[12] Kiolbassa K, Miksch A, Hermann K et al. Becoming a general practitioner-which factors have most impact on career choice of medical students? BMC Fam Pract 2011; 12: 25

[13] Natanzon I, Szecsenyi J, Ose D et al. Future potential country doctor: the perspectives of German GPs. Rural Remote Health 2010; 10: 1347

[14] Mayring P. Qualitative Inhaltsanalyse. Grundlagen und Techniken. 12. Aufl Weinheim/Basel: Beltz; 2015

[15] Blanz M. Forschungsmethoden und Statistik für die Soziale Arbeit: Grundlagen und Anwendungen. Stuttgart: Kohlhammer; 2015

[16] Böhme K, Kotterer A, Simmenroth-Nayda A. Allgemeinmedizin im Praktischen Jahr - eine Lösung für Nachwuchsprobleme in der hausärztlichen Versorgung? Z Allg Med 2013; 89: 452-458

[17] Birck S, Gedrose B, Robra BP et al. Stabilität der beruflichen Endziele im Verlauf der fachärztlichen Weiterbildung. Ergebnisse einer multizentrischen Kohortenstudie mit zweijährigem Intervall. Dtsch Med Wochenschr 2014; 139: 2173-2177

[18] Bundesministerium für Bildung und Forschung. Masterplan Medizinstudium 2020 (31.03.2017). Im Internet (Stand: 30.07.2018): https://www. bmbf.de/files/2017-03-31_Masterplan\%20Beschlusstext.pdf

[19] Barth N, Linde K, Schneider A. Niederlassungsmotive - Die Bereitschaft zur Niederlassung in eigener von Ärztinnen und Ärzten in Weiterbildung zum Facharzt für Allgemeinmedizin. Gesundheitswesen 2017; 79: 638644

[20] Broermann M, Messermaker A, Pauscher I et al. Spät entschieden und jetzt Allgemeinmedizin: Über Umwege zur gemeinschaftlichen Hausarztpraxis. Z Allg Med 2017; 93: 324-328

[21] Gibis B, Heinz A, Jacob R et al. Berufserwartungen von Medizinstudierenden. Ergebnisse einer bundesweiten Befragung. Dtsch Arztebl Int 2012; 109: 327-332 
[22] Steinhaeuser ], Otto P, Goetz K et al. Rural area in a European country from a health care point of view: an adoption of the Rural Ranking Scale. BMC Health Serv Res 2014; 14: 147

[23] Maenner M, Schneider D, Schaffer S et al. Welche Argumente motivieren für eine Landarzttätigkeit. Z Allg Med 2015; 91: 154-159

[24] Tamayo M. Generation X, Y, Z ... Viel zu viel Klischee. Dtsch Arzteb 2015; 112: A-17

[25] Barthen L, Gerlinger T. Die hausärztliche Versorgungssituation in Sachsen-Anhalt. Wahrnehmung, Beschreibung und Bewertung lokaler Versorgungsprobleme durch die Bürgermeister. Gesundheitswesen 2016; 78: $645-650$

[26] Stock C, Szecsenyi ], Riedinger-Riebl U et al. Projektion des Bedarfs an hausärztlicher Versorgung auf Gemeindeebene. Gesundheitswesen 2015; 77: 939-946

[27] Scheidt LR, Joos S, Szecsenyi ] et al. Überversorgt? Unterversorgt? - die Sicht von Bürgermeistern in Baden-Württemberg: Ein Beitrag zur Diskussion um die wohnortnahe medizinische Versorgung. Gesundheitswesen 2015; 77: e179-e183
[28] Steinhauser ], Scheidt L, Szecsenyi ] et al. Die Sichtweise der kommunalen Ebene über den Hausärztemangel - eine Befragung von Bürgermeistern in Baden-Württemberg. Gesundheitswesen 2012; 74: 612617

[29] Ulrich LR, Pham TT, Gerlach FM et al. Family Health Teams in Ontario Vorstellung eines kanadischen Primärversorgungsmodells und Anregungen für Deutschland. Gesundheitswesen 2019; 81: 492-497

[30] Tandjung R, Garaventa-Tadres D, Rosemann T et al. Spezifische Weiterbildungsangebote für Hausarztmedizin in der Schweiz - Bestandsaufnahme verschiedener kantonaler Programme. Praxis (Bern) 2013; 102: 843-849

[31] Terschuren C, Mensing M, Mekel OC. Is telemonitoring an option against shortage of physicians in rural regions? Attitude towards telemedical devices in the North Rhine-Westphalian health survey, Germany. BMC Health Serv Res 2012; 12: 95 